\title{
The $G C K R$ rs780094 polymorphism is associated with elevated fasting serum triacylglycerol, reduced fasting and OGTT-related insulinaemia, and reduced risk of type 2 diabetes
}

T. Sparsø • G. Andersen • T. Nielsen • K. S. Burgdorf •

A. P. Gjesing • A. L. Nielsen • A. Albrechtsen •

S. S. Rasmussen • T. Jørgensen • K. Borch-Johnsen •

A. Sandbæk • T. Lauritzen • S. Madsbad • T. Hansen •

O. Pedersen

Received: 22 June 2007 / Accepted: 28 September 2007 / Published online: 16 November 2007

(C) Springer-Verlag 2007

\begin{abstract}
Aims/hypothesis Recent genome-wide association studies have suggested that a polymorphism in $G C K R$, the gene encoding the glucokinase regulatory protein, is involved in
\end{abstract}

T. Sparsø and G. Andersen contributed equally to this study.

Electronic supplementary material The online version of this article (doi:10.1007/s00125-007-0865-z) contains supplementary material, which is available to authorised users.

T. Sparsø $(\varangle) \cdot$ G. Andersen $\cdot$ T. Nielsen $\cdot$ K. S. Burgdorf $•$

A. P. Gjesing • A. L. Nielsen $\cdot$ S. S. Rasmussen •

K. Borch-Johnsen $\cdot$ T. Hansen $\cdot$ O. Pedersen

Steno Diabetes Center, Niels Steensens Vej 1,

NLC2.13, DK-2820 Gentofte, Denmark

e-mail: tspr@steno.dk

A. Albrechtsen

Department of Biostatistics, University of Copenhagen,

Copenhagen, Denmark

T. Jørgensen $\cdot \mathrm{K}$. Borch-Johnsen

Research Centre for Prevention and Health,

Glostrup University Hospital,

Glostrup, Denmark

K. Borch-Johnsen • O. Pedersen

Faculty of Health Science, University of Aarhus,

Aarhus, Denmark

A. Sandbæk $\cdot$ T. Lauritzen

Department of General Practice, University of Aarhus,

Aarhus, Denmark

S. Madsbad

Department of Epidemiology, Hvidovre University Hospital,

Hvidovre, Denmark triacylglycerol regulation. Our aim was to examine in largescale studies the common GCKR rs780094 polymorphism in relation to metabolic traits (mainly fasting hypertriacylglycerolaemia) and traits related to pancreatic beta cell function.

Methods The polymorphism was genotyped in 16,853 Danes using Taqman allelic discrimination. Association was analysed in case-control studies and quantitative trait analyses. We also analysed the possible interactive effect between the $G C K-30 \mathrm{G}>\mathrm{A}$ polymorphism and the $G C K R$ rs780094 variant on metabolic traits.

Results The minor GCKR A-allele of rs780094 is associated with an increased level of fasting serum triacylglycerol $\left(p=6 \times 10^{-14}\right)$, impaired fasting $(p=0.001)$ and OGTTrelated insulin release $\left(p=3 \times 10^{-6}\right)$, reduced homeostasis model assessment of insulin resistance $(p=0.0004)$, WHOdefined dyslipidaemia $\left(p=6 \times 10^{-9}\right)$ and a modestly decreased risk of type 2 diabetes $(p=0.01)$. Significantly increased fasting serum insulin concentrations were demonstrated when analysing the GCK $-30 \mathrm{~A}$ and GCKR rs780094 G-alleles in an additive model.

Conclusions/interpretation The GCKR rs780094 polymorphism, or another variant with which it is in tight linkage disequilibrium, is likely to increase glucokinase regulatory protein activity to induce improved glycaemic regulation at the expense of hypertriacylglycerolaemia as reflected in the present study of 16,853 Danes. We also suggest an additive effect of $G C K$ and $G C K R$ risk alleles on plasma glucose and serum insulin release.

Keywords Association - Genetics · Genome-wide association · Glucokinase · Triacylglycerol · Type 2 diabetes 


$\begin{array}{ll}\text { Abbreviations } \\ \text { GK } & \text { glucokinase } \\ \text { GKRP } & \begin{array}{l}\text { glucokinase regulatory protein } \\ \text { genome-wide association } \\ \text { GWA }\end{array} \\ \text { HOMA-IR } & \begin{array}{l}\text { homeostasis model assessment of insulin } \\ \text { resistance } \\ \text { minor allele frequency }\end{array} \\ \text { MAF } & \begin{array}{l}\text { single-nucleotide polymorphism } \\ \text { SNP }\end{array}\end{array}$

\section{Introduction}

Liver and beta cell glucokinase (GK) plays a key role in the regulation of blood glucose homeostasis by enhancing insulin secretion from the pancreatic beta cells and by regulating glucose utilisation and production in the liver [1]. GK is regulated by the glucokinase regulatory protein (GKRP), a process depending on fructose 6-phosphate and fructose 1-phosphate [2]. The relationship between GK and diabetes mellitus has been demonstrated by the identification of specific rare mutations in $G C K$ (the gene encoding GK) as the cause of subsets of maturity-onset diabetes of the young [3] and permanent neonatal diabetes mellitus [4]. Similarly, a common $-30 \mathrm{G}>\mathrm{A}$ promoter polymorphism of $G C K$ increases the risk of hyperglycaemia in the general population [5]. The GCKR gene encodes GKRP and is localised to chromosome $2 \mathrm{p} 23.2-3$ [6], a genomic region previously linked to metabolic traits like fat mass and circulating lipid concentrations [7]. Gckr-deficient mice display reduced production of hepatic GK and exhibit impaired postprandial glycaemic control, albeit with no noteworthy loss in insulin secretion or changes in fasting blood glucose concentrations $[8,9]$. Adenoviral-mediated hepatic overproduction of GKRP significantly improved fasting and glucose-stimulated glycaemia in mice and resulted in a concomitant increase in insulin sensitivity, decreased leptin concentrations and increased triacylglycerol levels [10]. Previous studies of GCKR have attempted to relate frequent genetic variation to metabolic phenotypes; however, results have until recently been either negative or performed in insufficient study samples [11, 12]. In a study of 57 unrelated French obese participants from families with linkage to chromosome $2 \mathrm{p} 21-23$, mutation detection resulted in the identification of three rare amino acid substitutions in addition to a known common Pro446Leu substitution and an intronic polymorphism [12]. None of the rare variants showed co-segregation with obesity. Moreover, in a casecontrol study of 720 obese and 384 lean participants, no difference in allele or genotype frequencies of the Pro446Leu polymorphism was detected [12]. By contrast, a tendency towards increased postprandial plasma insulin concentrations among codon 446 Leu carriers was observed in 520 Danish twins, although this study was of a limited size [11].
Recent advances in genotyping techniques have facilitated the use of genome-wide association (GWA) studies in thorough investigations of the genetic background underlying common polygenic and multifactorial traits of the metabolic syndrome [13-19]. Such GWA studies have confirmed several known or putative type 2 diabetes genes such as PPARG, KCNJ11 and TCF7L2 [13, 15, 16, 18, 19], and have provided several new and promising insights represented by variation in SLC30A8, CDKAL1, HHEX, $C D K N 2 A, C D K N 2 B$ and IGF2BP2 [13, 15, 16, 18, 19]. Some of these novel candidate genes have no or limited known biological function and both validation of trait association and genotype-physiology studies of such novel findings are crucial. Initial GWA studies of type 2 diabetes and related metabolic traits have revealed a strong association of the GCKR gene polymorphism rs780094 (minor A-allele) with hypertriacylglycerolaemia $[13,15]$. Curiously, the investigators also observed modestly decreased plasma glucose levels, increased insulin sensitivity as estimated by the homeostasis model assessment of insulin resistance (HOMA-IR) and lower risk of type 2 diabetes [13]. Here, we sought to validate the reported associations between the GCKR rs780094 polymorphism and hypertriacylglycerolaemia. We further explored the relationship of this variant with extensive metabolic phenotypes in a large study of Danish white participants. Additionally, we tried to identify possible interactive effects between the GCKR rs780094 and $G C K-30 \mathrm{G}>\mathrm{A}$ polymorphisms.

\section{Methods}

Study population The GCKR rs780094 polymorphism was genotyped in 16,853 Danes comprising: (1) the populationbased Inter99 sample of middle-aged Danes sampled at Research Centre for Prevention and Health $(n=5,897)$ [20]; (2) type 2 diabetic patients sampled through the outpatient clinic at Steno Diabetes Center $(n=2035)$; (3) a population-based group of middle-aged glucose-tolerant participants recruited by Steno Diabetes Center $(n=504)$; and (4) the ADDITION study group sampled through Department of General Practice at University of Aarhus $(n=8,417)[21]$. Detailed descriptions of study populations are available in the Electronic supplementary material (ESM; ESM Table 1).

Study groups 1 and 3 underwent a standard 75 g OGTT. Informed written consent was obtained from all participants before participation. The study was approved by the Ethical Committee of Copenhagen County and was in accordance with the principles of the Helsinki Declaration. Type 2 diabetes, obesity and dyslipidaemia were defined according to WHO [22]. 
Biochemical and anthropometrical measurements Height and body weight were measured in light indoor clothes and without shoes; BMI was calculated as weight $(\mathrm{kg}) /[$ height $(\mathrm{m})]^{2}$. Waist circumference was measured in the standing position, midway between the iliac crest and the lower costal margin; hip circumference was measured at its maximum. Blood samples were drawn after a $12 \mathrm{~h}$ overnight fast. Plasma glucose was analysed by a glucose oxidase method (Granutest; Merck, Darmstadt, Germany). $\mathrm{HbA}_{1 \mathrm{c}}$ was measured by ion-exchange high performance liquid chromatography (normal reference range: 4.1-6.4\%) and serum insulin, excluding $\operatorname{des}(31,32)$ and intact proinsulin, was measured using an insulin kit (AutoDELFIA; Perkin-Elmer/Wallac, Turku, Finland). Serum Cpeptide concentrations were measured by a time-resolved fluoroimmunoassay (AutoDELFIA C-peptide kit; PerkinElmer/Wallac). Serum triacylglycerol, total cholesterol and HDL-cholesterol were analysed using enzymatic colorimetric methods (GPO-PAP and CHOD-PAP; Roche Molecular Biochemicals, Basel, Switzerland). HOMA-IR was calculated as described in [23]. (BIGTT-S $\mathrm{S}_{\mathrm{I}}$ ) and (BIGTT-AIR) were calculated as described [24].

Genotyping The GCKR rs780094 polymorphism was genotyped using Taqman allelic discrimination (KBioscience, Hoddesdon, UK). Discordance between 1,464 random duplicate samples was $0.3 \%$ and the genotyping success rate was $98 \%$. All genotype groups obeyed Hardy-Weinberg equilibrium. Genotyping of the $G C K-30 \mathrm{G}>\mathrm{A}$ polymorphism (rs1799884) has been previously described [5].

Statistical analysis Fisher's exact test was applied to examine differences in allele frequencies and genotype distributions between affected and unaffected participants. A general linear model was used to test quantitative variables for differences between genotype groups among glucose-tolerant and untreated participants. In order to assess the combined effect of two single-nucleotide polymorphisms (SNPs) in $G C K$ and $G C K R$, we used a linear model assuming equal effects of each risk allele at the two loci and compared this with a model lacking the risk allele parameter. However, in order to make such assumptions, we first tested whether the effect size of each allele was equal. All analyses were performed using SPSS version 14.0 (Chicago, IL, USA and RGui version 2.5.0 (http://www.r-project.org/). A $p$ value of less than 0.05 was considered to be significant.

\section{Results}

The rs780094 was genotyped in a total of 16,853 Danes and had an overall minor allele frequency (MAF) of $34.7 \%$.
We analysed quantitative metabolic traits reflecting fasting and glucose-stimulated levels of serum insulin, serum Cpeptide and plasma glucose $(0,30$ and 120 min during an OGTT) as well as fasting serum lipids (triacylglycerol, total cholesterol and HDL-cholesterol) in the population-based Inter99 study sample of 5,780 individuals with normal glucose tolerance $(74 \%)$, impaired fasting glucose $(8 \%)$, impaired glucose tolerance $(11 \%)$ or screen-detected, treatment-naive type 2 diabetes $(6 \%)$. The rs780094 minor A-allele was highly associated with reduced fasting ( $p=$ $0.001), 30 \mathrm{~min}\left(p=1.3 \times 10^{-7}\right)$ and $120 \mathrm{~min}(p=0.03)$ postOGTT insulin release (Table 1). Concomitantly, reduced fasting, $30 \mathrm{~min}$ and $120 \mathrm{~min}$ serum C-peptide concentrations were demonstrated, as well as increased insulin sensitivity as assessed by HOMA-IR $(p=0.0004)$ and BIGTT-S I $(p=0.01$; Table 1). Accordingly, a significant decrease was observed in the incremental AUC for serum C-peptide and insulin during an OGTT in participants homozygous for the minor A-allele. No considerable relationship was observed with levels of plasma glucose (fasting, $30 \mathrm{~min}$ or $120 \mathrm{~min}$ post-OGTT) or the incremental AUC for glucose (Table 1). For fasting serum triacylglycerol we observed a major increase in concentrations related to the rs780094 A-allele $\left(p=6 \times 10^{-14}\right)$ and this was accompanied by a minor elevation of total fasting serum total cholesterol $(p=0.03)$.

Three case-control studies of rs 780094 were performed in relation to type 2 diabetes, BMI-defined obesity and dyslipidaemia. The polymorphism was associated with type 2 diabetes $(p=0.02$ for genotype distribution and $p=0.01$ for MAF; Table 2). In a second case-control study the participants were stratified according to their BMI levels; however, we did not observe any differences in MAF or genotype distribution between individuals with a $\mathrm{BMI}<$ $25 \mathrm{~kg} / \mathrm{m}^{2}$ and those with a BMI>30 kg/m ${ }^{2}$ (Table 2). Finally, we investigated the impact of the rs780094 genotypes on healthy normolipidaemic individuals as opposed to participants with WHO-defined dyslipidaemia. Highly significant differences were observed in the MAF [33.3\% (95\% CI $32.4-34.2 \%)$ vs $38.3 \%$ (36.9-39.8\%), $\left.p=6 \times 10^{-9}\right]$ and the distribution of genotypes $\left(p=6 \times 10^{-9}\right)$ between the two groups (Table 2).

We investigated the additive effect of GCKR rs780094 and $G C K-30 \mathrm{G}>\mathrm{A}$ on fasting and post-OGTT concentrations of plasma glucose and serum insulin in the Inter99 study sample, assuming that the rs780094 G-allele and -30 A-allele were the risk alleles (Fig. 1). We observed a significantly increased fasting serum insulin concentration in a model including both of the two SNPs $(p=0.0002$ for additivity) when compared with the null model (no SNPs added). The additive model included one additive covariate $\left(X_{\text {add }}=0,1,2,3,4\right.$; for each risk allele of the two SNPs). A test for non-linear interaction (epistasis) between the two SNPs was negative. 
Table 1 Anthropometric and metabolic characteristics of 5,780 treatment-naïve Danish participants (Inter99) stratified according to GCKR rs780094 genotype

\begin{tabular}{|c|c|c|c|c|c|c|}
\hline Characteristics & GG & GA & AA & $p_{\mathrm{a}}$ value & $p_{\mathrm{d}}$ value & $P_{\mathrm{r}}$ value \\
\hline$n$ (men/women) & $2,461(1,209 / 1,252)$ & $2,617(1,320 / 1,297)$ & $702(349 / 353)$ & & & \\
\hline Age (years) & $46 \pm 8$ & $46 \pm 8$ & $46 \pm 8$ & & & \\
\hline BMI $\left(\mathrm{kg} / \mathrm{m}^{2}\right)$ & $26.2 \pm 4.5$ & $26.2 \pm 4.5$ & $26.2 \pm 4.6$ & 0.9 & 0.9 & 0.7 \\
\hline \multicolumn{7}{|c|}{ Fasting serum lipids (mmol/l) } \\
\hline Triacylglycerol & $1.27 \pm 1.50$ & $1.32 \pm 0.98$ & $1.59 \pm 1.90$ & $6 \times 10^{-14}$ & $1 \times 10^{-8}$ & $6 \times 10^{-12}$ \\
\hline Total cholesterol & $5.5 \pm 1.0$ & $5.6 \pm 1.1$ & $5.6 \pm 1.1$ & 0.03 & 0.03 & 0.3 \\
\hline HDL-cholesterol & $1.4 \pm 0.4$ & $1.4 \pm 0.4$ & $1.4 \pm 0.4$ & 0.08 & 0.1 & 0.2 \\
\hline \multicolumn{7}{|c|}{ Plasma glucose $(\mathrm{mmol} / \mathrm{l})$} \\
\hline Fasting & $5.6 \pm 0.8$ & $5.5 \pm 0.8$ & $5.5 \pm 0.9$ & 0.008 & 0.02 & 0.07 \\
\hline $30 \mathrm{~min}$ post-OGTT & $8.7 \pm 1.8$ & $8.7 \pm 1.9$ & $8.6 \pm 2.0$ & 0.1 & 0.3 & 0.2 \\
\hline $120 \mathrm{~min}$ post-OGTT & $6.2 \pm 2.0$ & $6.2 \pm 2.1$ & $6.3 \pm 2.4$ & 0.2 & 0.4 & 0.1 \\
\hline $\mathrm{AUC}(\mathrm{mmol} / 1 \times \min )$ & $219 \pm 133$ & $221 \pm 136$ & $225 \pm 139$ & 0.3 & 0.5 & 0.3 \\
\hline \multicolumn{7}{|l|}{ Serum insulin $(\mathrm{pmol} / \mathrm{l})$} \\
\hline Fasting & $35(24-52)$ & $34(23-50)$ & $32(23-49)$ & 0.001 & 0.003 & 0.03 \\
\hline 30 min post-OGTT & $256(181-369)$ & $238(174-342)$ & $232(167-333)$ & $1 \times 10^{-7}$ & $4 \times 10^{-7}$ & 0.001 \\
\hline 120 min post-OGTT & $158(98-261)$ & $155(96-249)$ & $154(90-242)$ & 0.03 & 0.1 & 0.06 \\
\hline $\mathrm{AUC}(\mathrm{pmol} / 1 \times \min )$ & $19,400(13,640-28,940)$ & $18,160(12,800-26,140)$ & $18,120(12,140-26,240)$ & $3 \times 10^{-6}$ & $2 \times 10^{-5}$ & 0.002 \\
\hline \multicolumn{7}{|c|}{ Serum C-peptide (pmol/1) } \\
\hline Fasting & $602 \pm 272$ & $595 \pm 276$ & $577 \pm 264$ & 0.002 & 0.02 & 0.004 \\
\hline $30 \mathrm{~min}$ post-OGTT & $2,042 \pm 739$ & $1,985 \pm 708$ & $1,920 \pm 659$ & $1 \times 10^{-5}$ & 0.0001 & 0.001 \\
\hline $120 \mathrm{~min}$ post-OGTT & $2,317 \pm 1,011$ & $2,315 \pm 1,021$ & $2,251 \pm 1,001$ & 0.09 & 0.4 & 0.02 \\
\hline $\operatorname{AUC}(\mathrm{pmol} / 1 \times \min )$ & $163,519 \pm 58,812$ & $160,476 \pm 57,443$ & $155,907 \pm 54,927$ & $1 \times 10^{-4}$ & 0.001 & 0.002 \\
\hline HOMA-IR & $8.6(5.8-13.0)$ & $8.2(5.5-12.8)$ & $7.8(5.3-12.3)$ & 0.0004 & 0.001 & 0.01 \\
\hline BIGTT-S $_{\text {I }}$ & $9.0(6.0-11.9)$ & $9.3(6.5-12.2)$ & $9.7(6.7-12.2)$ & 0.01 & 0.01 & 0.2 \\
\hline BIGTT-AIR & $1,639(1,296-2,097)$ & $1,606(1,261-2,067)$ & $1,664(1,314-2,051)$ & 0.04 & 0.02 & 0.5 \\
\hline
\end{tabular}

Data are means \pm SD or median (interquartile range)

Values of serum insulin, values derived from insulin variables and values of serum triacylglycerol were logarithmically transformed before statistical analysis. All analyses were made using additive, dominant and recessive models

Calculated $p$ values were adjusted for age, sex and BMI (where appropriate); genotype and sex were considered as fixed factors and age and BMI as covariates

AUC, incremental area under the curve

\section{Discussion}

In the present large population-based study of treatmentnaïve middle-aged people in Inter99 we validated the recent report $[13,15]$ of a relationship between the minor A-allele of the GCKR rs780094 polymorphism and increased fasting serum triacylglycerol concentrations. Further studies of quantitative variables of metabolism showed that carriers of the minor A-allele had a substantially lower serum insulin release and were more insulin-sensitive. In statistically powered case-control studies involving 7,619 middle-aged participants, we demonstrated that the variant conferred an increased risk of WHO-defined dyslipidaemia and a modestly lower risk of type 2 diabetes. We failed to demonstrate any significant association with obesity.

Improved glycaemic regulation at the expense of hypertriacylglycerolaemia and other abnormalities in energy metabolism has been demonstrated in mice and rat liver overproducing either GK or GKRP $[10,25]$. GKRP is produced in molar excess over GK and the regulation of GK by GKRP may be very sensitive to changes in tissue levels of GKRP [6]. Thus, we speculate that the GCKR rs780094 polymorphism, or another variant with which it is in tight linkage disequilibrium, influences GKRP production levels and thereby induces an increased function of GKRP. The observed decrease in serum insulin release among GCKR rs780094 carriers may lead to a secondary increase in serum triacylglycerol due to increased hepatic fatty acid oxidation, while the $G C K-30 \mathrm{G}>\mathrm{A}$ polymorphism influencing beta cell-specific GK production may act to increase hyperglycaemia by elevating the threshold for glucose-stimulated insulin release. In studies of the general middle-aged population the $G C K-30 \mathrm{G}>\mathrm{A}$ polymorphism has previously shown a graded relationship with hyperglycaemia [5]. Therefore, we examined the possible twoway epistasis of this variant and the GCKR rs780094 polymorphism on plasma glucose and serum insulin concentrations in the Inter99 study sample, but failed to 
Table 2 Genotype distribution and MAF for the GCKR rs780094 polymorphism in participants stratified according to glucose tolerance status, BMI levels and dyslipidaemia

\begin{tabular}{|c|c|c|c|c|c|c|}
\hline & $n$ (men/women) & GG & GA & AA & MAF $(95 \% \mathrm{CI})$ & OR $(95 \% \mathrm{CI})$ \\
\hline \multicolumn{7}{|c|}{ Glucose tolerance status } \\
\hline NGT & $4,891(2,272 / 2,619)$ & $2,066(42)$ & $2,234(46)$ & $591(12)$ & $34.9(34.0-35.9)$ & \\
\hline Diabetes & $3,878(2,302 / 1,573)$ & $1,755(45)$ & $1,681(43)$ & $442(12)$ & $33.1(32.1-34.2)$ & \\
\hline$p_{\mathrm{GD}}$ value & & & 0.02 & & & $0.92(0.87-0.98)$ \\
\hline$p_{\mathrm{MAF}}$ value & & & & & 0.01 & \\
\hline \multicolumn{7}{|l|}{ BMI status } \\
\hline $\mathrm{BMI}<25 \mathrm{~kg} / \mathrm{m}^{2}$ & $5,423(2,270 / 3,153)$ & $2,358(44)$ & $2,442(45)$ & $623(12)$ & $34.0(33.1-34.9)$ & \\
\hline $\mathrm{BMI} \geq 30 \mathrm{~kg} / \mathrm{m}^{2}$ & $4,909(2,535 / 2,374)$ & $2,161(44)$ & $2,183(45)$ & $565(12)$ & $33.7(32.8-34.7)$ & \\
\hline$p_{\mathrm{GD}}$ value & & & 0.8 & & & $0.99(0.93-1.05)$ \\
\hline$p_{\mathrm{MAF}}$ value & & & & & 0.7 & \\
\hline \multicolumn{7}{|l|}{ Lipid status } \\
\hline Normolipidaemia & $5,415(2,437 / 2,978)$ & $2,385(44)$ & $2,450(45)$ & $580(11)$ & $33.3(32.4-34.2)$ & \\
\hline Dyslipidaemia & $2,204(1,378 / 826)$ & $852(39)$ & $1,014(46)$ & $338(15)$ & $38.3(36.9-39.8)$ & \\
\hline$p_{\mathrm{GD}}$ value & & & $6 \times 10^{-9}$ & & & $1.24(1.15-1.33)$ \\
\hline$p_{\mathrm{MAF}}$ value & & & & & $5 \times 10^{-9}$ & \\
\hline
\end{tabular}

Data are number of participants with each genotype (\% of each group); frequencies of the minor A-allele (MAF) are in percentages Dyslipidaemia was defined as serum triacylglycerol $\geq 1.7 \mathrm{mmol} / 1$ or HDL-cholesterol $<0.9 \mathrm{mmol} / 1$ for men or $<1.0 \mathrm{mmol} / 1$ for women or current or previous treatment with lipid-lowering drugs

All $p$ values were calculated using Fisher's exact test and compare genotype distributions $\left(p_{\mathrm{GD}}\right)$ and MAFs $\left(p_{\mathrm{MAF}}\right)$

NGT, normal glucose tolerance; OR, odds ratio

show any evidence of an interaction (data not shown). However, when combining the two risk alleles, we demonstrated a significant effect on fasting serum insulin concentrations in a model including an additive covariate. Even though the two specific gene products may act in different tissues, polymorphisms in GCK and GCKR possibly combine to further increase the risk of hyperglycaemia compared with the individual risk variants.

Although we observed a large effect of the rs780094 on serum insulin concentrations and a modest effect size on type 2 diabetes compared with persons with normal glucose tolerance, we were somewhat surprised that we were unable to find a significant effect on plasma glucose levels at 30 and 120 min post-OGTT; however, it seems that the effect of

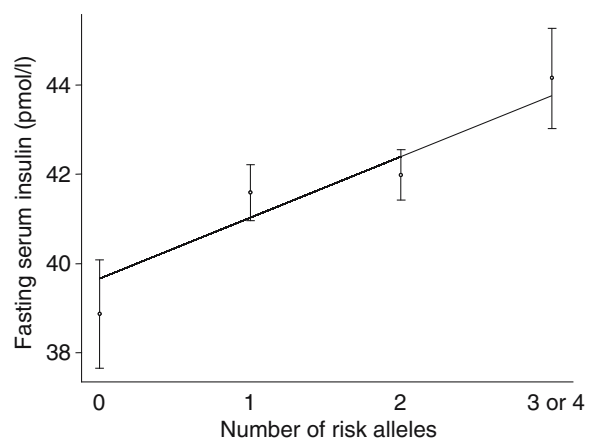

Fig. 1 Additive effect of the $G C K R$ rs780094 and $G C K-30 \mathrm{G}>\mathrm{A}$ variants on fasting serum insulin. Values are means $\pm \mathrm{SD}$. Risk alleles are the rs780094 G-allele and $-30 \mathrm{~A}$-allele. $p=0.0002$ for the two SNPs (estimated) rs780094 on serum insulin may not be sufficient to influence plasma glucose levels. Moreover, the fact that glucose levels are regulated by various compensatory mechanisms may also explain this observation. In addition, the polymorphism in GCKR seems to associate with type 2 diabetes through reduced beta cell function and individuals carrying the risk allele may compensate for this reduction with a healthy life style.

According to the HapMap website (http://www.hapmap. org/index.html.en) the GCKR rs780094 polymorphism is located in a large haploblock spanning at least $500 \mathrm{~kb}$ and including several other known genes, which indeed questions the proposal that the intronic rs780094 is the causative SNP responsible for the observed metabolic changes. A thorough mutation analysis of the DNA region in question needs to be carried out, e.g. by nucleotide sequencing of selected individuals, in order to identify genetic variation that may explain to a larger extent the inter-individual variation in metabolic variables. In addition, the combined effect (such as an additive, epistatic or haplotypes) of other polymorphisms within the region or the regulatory pathway may contribute significantly to the observed differences in metabolic traits. Also, possible gene-environment interactions need to be considered and investigated [26].

Acknowledgements This study was supported by the Danish Medical Research Council, the Danish Diabetes Association, the Gerda and Aage Haensch Foundation, the A P Møller Foundation for the Advancement of Medical Science, the University of Copenhagen and the Velux Foundation. This work is part of the project 'Hepatic and 
adipose tissue and functions in the metabolic syndrome' (HEPADIP; www.hepadip.org), which is supported by the European Commission as an integrated project under the 6th Framework Programme (LSHMCT-2005-018734). The Danish Obesity Research Centre (DanORC; www.danorc.dk) is supported by The Danish Council for Strategic Research (grant 2101-06-0005). The ADDITION study has the ClinicalTrials.gov ID NCT00237548. The authors wish to thank A. Forman, I.-L. Wantzin and M. Stendal for technical assistance, and G. Lademann for secretarial support.

Duality of interest K. Borch-Johnsen holds stock in Novo Nordisk and has received lecture fees from pharmaceutical companies. The remaining authors declare that there is no duality of interest associated with this manuscript.

\section{References}

1. Matschinsky FM (1996) Banting lecture 1995: a lesson in metabolic regulation inspired by the glucokinase sensor paradigm. Diabetes 45:223-241

2. Van Schaftingen E (1989) A protein from rat liver confers to glucokinase the property of being antagonistically regulated by fructose 6-phosphate and fructose 1-phosphate. Eur J Biochem 179:179-184

3. Hattersley AT, Turner RC, Permutt MA et al (1992) Linkage of type 2 diabetes to the glucokinase gene. Lancet 339:1307-1310

4. Njølstad PR, Søvik O, Cuesta-Munoz A et al (2001) Neonatal diabetes mellitus due to complete glucokinase deficiency. N Engl J Med 344:1588-1592

5. Rose CS, Ek J, Urhammer SA (2005) A $-30 \mathrm{G}>$ A polymorphism of the $\beta$-cell-specific glucokinase promoter associates with hyperglycemia in the general population of whites. Diabetes 54:3026-3031

6. Hayward BE, Fantes JA, Warner JP (1996) Co-localization of the ketohexokinase and glucokinase regulator genes to a $500-\mathrm{kb}$ region of chromosome 2p23. Mamm Genome 7:454-458

7. Comuzzie AG, Hixson JE, Almasy L et al (1997) A major quantitative trait locus determining serum leptin levels and fat mass is located on human chromosome 2. Nat Genet 15:273-276

8. Farrelly D, Brown KS, Tieman A et al (1999) Mice mutant for glucokinase regulatory protein exhibit decreased liver glucokinase: a sequestration mechanism in metabolic regulation. Proc Natl Acad Sci U S A 96:14511-14516

9. Grimsby J, Coffey JW, Dvorozniak MT et al (2000) Characterization of glucokinase regulatory protein-deficient mice. J Biol Chem 275:7826-7831

10. Slosberg ED, Desai UJ, Fanelli B (2001) Treatment of type 2 diabetes by adenoviral-mediated overexpression of the glucokinase regulatory protein. Diabetes 50:1813-1820

11. Køster B, Fenger M, Poulsen P, Vaag A, Bentzen J (2005) Novel polymorphisms in the GCKR gene and their influence on glucose and insulin levels in a Danish twin population. Diabet Med 22:1677-1682

12. Veiga-da-Cunha M, Delplanque J, Gillain A et al (2003) Mutations in the glucokinase regulatory protein gene in $2 \mathrm{p} 23$ in obese French Caucasians. Diabetologia 46:704-711

13. Diabetes Genetics Initiative of Broad Institute of Harvard and MIT, LUND University, Novartis Institutes for BioMedical Research (2007) Genome-wide association analysis identifies loci for type 2 diabetes and triglyceride levels. Science 316:1331-1336

14. Frayling TM, Timpson NJ, Weedon MN et al (2007) A common variant in the FTO gene is associated with body mass index and predisposes to childhood and adult obesity. Science 316:889-894

15. Scott LJ, Mohlke KL, Bonnycastle LL et al (2007) A genomewide association study of type 2 diabetes in Finns detects multiple susceptibility variants. Science 316:1341-1345

16. Sladek R, Rocheleau G, Rung J (2007) A genome-wide association study identifies novel risk loci for type 2 diabetes. Nature 445:881-885

17. Steinthorsdottir V, Thorleifsson G, Reynisdottir I et al (2007) A variant in CDKAL1 influences insulin response and risk of type 2 diabetes. Nat Genet 39:770-775

18. Zeggini E, Weedon MN, Lindgren CM et al (2007) Replication of genome-wide association signals in UK samples reveals risk loci for type 2 diabetes. Science 316:1336-1341

19. The Wellcome Trust Case Control Consortium (2007) Genomewide association study of 14,000 cases of seven common diseases and 3,000 shared controls. Nature 447:661-678

20. Jørgensen T, Borch-Johnsen K, Thomsen TF, Ibsen H, Glümer C, Pisinger C (2003) A randomized non-pharmacological intervention study for prevention of ischaemic heart disease: baseline results Inter99 (1). Eur J Cardiovasc Prevention Rehab 10:377-386

21. Lauritzen T, Griffin S, Borch-Johnsen K et al (2000) The ADDITION study: proposed trial of the cost-effectiveness of an intensive multifactorial intervention on morbidity and mortality among people with type 2 diabetes detected by screening. Int $\mathrm{J}$ Obes Suppl 3:S6-S11

22. Alberti KGMM, Zimmett PZ for a WHO consultation (1999) Definition, diagnosis and classification of diabetes mellitus and its complications; Part 1: diagnosis and classification of diabetes mellitus. World Health Organization, Geneva

23. Matthews DR, Hosker JP, Rudenski AS et al (1985) Homeostasis model assessment: insulin resistance and $\beta$-cell function from fasting plasma glucose and insulin concentrations in man. Diabetologia 28:412-419

24. Hansen T, Drivsholm T, Urhammer SA et al (2007) The BIGTT test. A novel test for simultaneous measurement of pancreatic $\beta$ cell function, insulin sensitivity, and glucose tolerance. Diabetes Care 30:257-262

25. O'Doherty RM, Lehman DL, Telemaque-Potts S, Newgard CB (1999) Metabolic impact of glucokinase overexpression in liver. Lowering of blood glucose in fed rats is accompanied by hyperlipidemia. Diabetes 48:2022-2027

26. Grarup N, Andersen G (2007) Gene-environment interactions in the pathogenesis of type 2 diabetes and metabolism. Curr Opin Clin Nutr Metab Care 10:420-426 